\title{
Natural antibodies with the T15 idiotype may act in atherosclerosis, apoptotic clearance, and protective immunity
}

\author{
Peter X. Shaw, ${ }^{1}$ Sohvi Hörkkö, ${ }^{1}$ Mi-Kyung Chang, ${ }^{1}$ Linda K. Curtiss, ${ }^{2}$ Wulf Palinski, ${ }^{1}$ \\ Gregg J. Silverman, ${ }^{1}$ and Joseph L. Witztum ${ }^{1}$ \\ ${ }^{1}$ Department of Medicine, University of California, San Diego, La Jolla, California, USA \\ ${ }^{2}$ The Scripps Research Institute, La Jolla, California, USA \\ Address correspondence to: Joseph L. Witztum, Department of Medicine, University of California, San Diego, 9500 Gilman \\ Drive, La Jolla, California 92093, USA. Phone: (858) 534-4347; Fax: (858) 534-2005; E-mail: jwitztum@ucsd.edu. \\ The laboratories of Gregg J. Silverman and Joseph L. Witztum contributed equally to this work. \\ Received for publication September 17, 1999, and accepted in revised form May 2, 2000.
}

The immune response to oxidized LDL (OxLDL) may play an important role in atherogenesis. Working with apoE-deficient mice, we isolated a panel of OxLDL-specific B-cell lines that secrete IgM Abs that specifically bind to oxidized phospholipids such as 1-palmitoyl-2-(5-oxovaleroyl)sn-glycero-3-phosphorylcholine (POVPC). These Abs block uptake of OxLDL by macrophages, recognize similar oxidation-specific epitopes on apoptotic cells, and are deposited in atherosclerotic lesions. The Abs were found to be structurally and functionally identical to classic "natural" T15 anti-PC Abs that are of B-1 cell origin and are reported to provide optimal protection from virulent pneumococcal infection. These findings suggest that there has been natural selection for B-1 cells secreting oxidation-specific/T15 antibodies, both for their role in natural immune defense and for housekeeping roles against oxidation-dependent neodeterminants in health and disease.

J. Clin. Invest. 105:1731-1740 (2000).

\section{Introduction}

Atherosclerosis is a chronic inflammatory disease that results from hyperlipidemia and a complex interplay of environmental, metabolic, and genetic risk factors. There is much evidence that the oxidation of LDL plays a central, if not obligatory, role in the atherogenic process (1). In addition, oxidation of LDL generates a variety of oxidatively modified lipids and proteins that represent highly immunogenic neodeterminants for the immune system. In murine models of atherosclerosis, such as apoE-deficient $\left(A p o E^{-/-}\right)$mice, atherosclerosis is correlated with the development of high titers of autoantibodies to various oxidation-specific neoepitopes of oxidized LDL (OxLDL) $(2,3)$. The consequences of such cellular and humoral responses are still poorly understood, but under certain conditions they can clearly modify the natural history of the disease. For example, immunization of hypercholesterolemic rabbits or mice with homologous LDL containing epitopes of OxLDL ameliorates atherosclerosis $(4,5)$.

To investigate the origins of these atherosclerosisassociated autoantibodies, we recently isolated a panel of B-cell hybridomas with specificity for OxLDL neodeterminants from the spleens of diseased $A p o E^{-/-}$mice that had not received experimental immunization or in vitro stimulation (3). Reflecting the disease-associated immune response, a minimum of $32 \%$ of $1,536 \mathrm{Ig}$ - secreting hybridomas expressed Abs that bound to one or more epitopes of OxLDL, of which 17 hybridoma lines were cloned and their secreted Abs termed "EO" Abs. All were IgMs that bound strongly to either a model epitope of Ox-LDL, malondialdehyde-modified LDL (MDA-LDL), or to LDL that was oxidized by exposure to copper (Cu-OxLDL) (3). Each of the Abs selected for binding to Cu-OxLDL was later shown to bind to oxidized phospholipids, and specifically to 1-palmitoyl-2-(5-oxovaleroyl)-sn-glycero-3-phosphorylcholine (POVPC), an oxidation product derived from 1-palmitoyl-2-arachidonyl-sn-glycero-3-phosphorylcholine (PAPC). (Please note that in this article PC stands for phosphorylcholine, not for phosphatidylcholine, an intact phospholipid containing two fatty acid chains and the phosphorylcholine headgroup.) Of considerable interest is the fact that all of these POVPC-specific autoantibodies recognized the lipid moiety of OxLDL, as well as the delipidated modified apoB (the protein moiety of OxLDL), but not native LDL. Thus, these Abs bind to oxidized phospholipids or their protein adducts (e.g., with apoB or BSA). In addition, each of these Abs was able to block macrophage scavenger receptor-mediated binding and uptake of OxLDL. POVPC (as a BSA adduct) also blocked the uptake of OxLDL (6). Macrophage scavenger receptors involved in uptake of OxLDL are also believed to be responsible for the recognition of apoptotic cells, as well as dam- 
aged or senescent cells. We demonstrated that the POVPC-specific EO Abs also bound to apoptotic cells and inhibited their phagocytosis by elicited peritoneal macrophages. Similarly, POVPC-BSA also inhibited the phagocytosis of apoptotic cells by macrophages (7). These data provide compelling evidence that these naturally arising autoantibodies bind to common oxidation-specific epitopes on both OxLDL and apoptotic cells that mediate macrophage recognition.

To gain a better understanding of the roles these IgM Abs play in health and disease, we investigated their genetic and structural origin. Our studies suggest that these anti-OxLDL autoantibodies represent highly conserved natural Abs that arise spontaneously in the perinatal period, and later become further selected in $A p o E^{-/-}$mice in response to the extensive burden of oxidation-specific antigens that occur with the development of atherosclerosis.

\section{Methods}

Antigens and ligands. Phosphorylcholine chloride (PC-Cl) was obtained from Sigma Chemical Co. (St. Louis, Missouri, USA). PC-conjugated keyhole limpet hemocyanin (PC-KLH) was from Biosearch Technologies Inc. (Novato, California, USA). PC-BSA and PC-histone were kind gifts of Norman Klinman (The Scripps Research Institute, La Jolla, California, USA) and Marvin Rittenberg (Oregon Health Sciences University, Portland, Oregon, USA), respectively. Purified pneumococcal cell-wall polysaccharide (C-PS) was obtained from the Staatenserum Institute (Copenhagen, Denmark). POVPC was synthesized, and POVPC-BSA adducts were generated in the presence of cyanoborohydride, as described (6). LDL was prepared in the presence of EDTA, and copper-oxidized LDL (Cu-OxLDL) and MDA-LDL were prepared as described (3).

Hybridomas and $m A b$ 's. Monoclonal autoantibodies directed against oxidation-specific epitopes of LDL were produced from hybridomas generated from apoE-deficient mice, which have very high titers of autoantibodies to $\mathrm{Cu}-\mathrm{OxLDL}$ (3). In brief, B lymphocytes from the spleens of two apoE-deficient mice that had not been immunized exogenously were fused with the P3 $\times$ 63Ag8.653.1 myeloma cell line. Hybridomas were screened for binding to model epitopes of OxLDL, including $\mathrm{Cu}-\mathrm{OxLDL}$ and MDA-LDL. Seventeen hybridoma cell lines were isolated by limiting dilution, and the Abs expressed were designated EO autoantibodies EO1-EO17. All were isotyped as IgM and characterized as described $(3,6)$. Abs used in this report were purified from ascites fluid by fast protein liquid chromatography (FPLC). The T(EPC)15 murine monoclonal IgA anti-PC $\mathrm{Ab}$ (referred to hereafter as T15) was the kind gift of $\mathrm{H}$. Kohler (University of Kentucky, Lexington, Kentucky, USA). Anti-idiotypic Abs Tc54.6 and T139.2 were kind gifts of M. Scharff (Albert Einstein College of Medicine, Bronx, New York, USA). AB1-2, an anti-T15 idiotype Ab, was provided by J. Kearney (University of Alabama at Birmingham, Birmingham, Alabama, USA).
Chemiluminescent immunoassay. To coat wells, protein antigens were diluted in PBS containing $0.27 \mathrm{mM}$ EDTA; $50 \mu \mathrm{L}$ per well was applied to 96 -well white round-bottomed MicroFluor microtiter plates (DYNEX Technologies, Chantilly, Virginia, USA) and left overnight at $4^{\circ} \mathrm{C}$. The wells were washed three times with PBS, and blocked with PBS containing 1\% BSA (BSA-PBS) for 30 minutes. Fifty microliters of primary Abs, diluted with BSA-PBS, were added to wells in the presence and absence of competitors, and then incubated for 1 hour at room temperature. The wells were washed three times with PBS. Binding of primary $\mathrm{Ab}$ was detected with isotype-specific alkaline phosphatase-labeled (AP-labeled) goat anti-mouse Ig secondary Abs (Sigma Chemical Co.) in Tris-buffered saline (TBS) buffer containing $150 \mathrm{mM} \mathrm{NaCl}, 50 \mathrm{mM}$ Tris base, $0.27 \mathrm{mM}$ EDTA, and 1\% BSA. This was followed by a rinse with water and the addition of $25 \mu \mathrm{L}$ of 50\% LumiPhos 530 solution (Lumigen Inc., Southfield, Michigan, USA). In some experiments, the mAb EO6 was biotinylated as described previously (6) and added to antigen-coated wells, followed by the addition of AP-labeled NeutrAvidin (Pierce Chemical Co., Rockford, Illinois, USA). The light emissions were measured as relative light units (RLU) over $100 \mathrm{~ms}$ using a DYNEX Luminometer (DYNEX Technologies) (6).

Isolation and amplification of Ab genes. Total RNA was extracted from $10^{7}$ hybridoma cells using RNA STAT60 (Tel-Test Inc., Friendswood, Texas, USA) using the manufacturer's protocol. A Superscript II cDNA synthesis kit (GIBCO BRL, Gaithersburg, Maryland, USA) was then used to synthesize cDNA with oligo(dT) primer. Each PCR reaction contained $0.5 \mu \mathrm{g}$ of cDNA template and $1 \mu \mathrm{g}$ of each oligonucleotide primer. The reactions were supplemented with dNTP, Taq polymerase (Pharmacia, Peapack, New Jersey, USA), and reaction buffer, and were amplified under optimized thermal cycler conditions. The oligonucleotide primers for PCR reactions were synthesized in the Molecular Biology Core facility of the La Jolla Specialized Center of Research for Molecular Medicine and Atherosclerosis. For the first round of PCR, heavy-chain variable $\left(V_{H}\right)$ genes were amplified in seven different reaction tubes, each containing one of a panel of seven sense primers targeted for the $\mathrm{V}_{\mathrm{H}}$ framework region 1 (FR1) subdomain, paired with an antisense primer specific for the $\mu$ constant region (CH1) (Table 1). The oligonucleotides were designed to amplify more than $80 \%$ of all expressed $\mathrm{V}_{\mathrm{H}}$ gene rearrangements (8). In preliminary studies, an immunoassay using anti- $\kappa$-specific $A b$ determined that each of the reported EO Abs uses a $\kappa$ light chain (not shown). Therefore, $V_{\kappa}$ gene rearrangements were amplified in six separate PCR reactions that included one of two degenerate $V_{\kappa}$ gene FR1 sense primers, and one of three antisense primers that each target distinct $\mathrm{J}_{\mathrm{K}}$ gene sequences (Table 1 ).

Sequence analysis of variable-region genes of EO bybridomas. The PCR products of the murine hybridoma $\mathrm{Ig} \mathrm{V}_{\mathrm{H}}$ and $V_{\kappa}$ gene rearrangements were purified after elec- 
trophoretic separation on a $2 \%$ agarose gel and then cloned into the TA cloning vector PCR2.1 (Invitrogen Corp., Carlsbad, California, USA), according to the manufacturer's protocol. Plasmid DNA from individual clones was purified using QIAprep columns (QIAGEN Inc., Chatsworth, California, USA), and the nucleotide sequences were determined with an ABI PRISM automated sequence analyzer (Perkin-Elmer Applied Biosystems, Foster City, California, USA) using T7 and M13 primers. The sequences were analyzed using software packages from the Genetics Computer Group (Madison, Wisconsin, USA) in combination with the EMBL/GenBank databases. Sequence alignments of the identified $V_{H}$ and $V_{\kappa}$ genes were also determined with BLAST tools of the National Center of Biotechnology Information (Bethesda, Maryland, USA).

Induction of apoptosis and flow cytometry analysis. Porcine aortic endothelial cells (PAECs) from wild-type swine were cultured in Medium 199 containing Earl's salt solution and $15 \% \mathrm{FCS}$ in a $\mathrm{CO}_{2}$-enriched atmosphere, as described elsewhere (7). Apoptosis was induced during the sixth passage by culturing PAECs for 16 hours in serum-free media, using Teflon ${ }^{\circledR}$ membrane-coated culture flasks (Sarstedt, Newton, New Jersey, USA) to inhibit anchorage-dependent cell spreading. Cells were then harvested and washed in ice-cold PBS with 1\% BSA. To determine Ab binding, $10^{6}$ cells were incubated with $50 \mu \mathrm{g} / \mathrm{mL}$ of T15 or isotype control murine IgA (from a myeloma cell line) at $4^{\circ} \mathrm{C}$ for 20 minutes; cells were then washed and incubated for another $20 \mathrm{~min}$ utes with $1 \mu \mathrm{g} / \mathrm{mL}$ of fluorescein-conjugated antimouse IgA (PharMingen, San Diego, California, USA). Cells were again washed and then incubated for $10 \mathrm{~min}$ utes with $1 \mu \mathrm{g} / \mathrm{mL}$ of PI. Data were immediately acquired with a FACScan instrument (Becton Dickinson and Co., Franklin Lakes, New Jersey, USA), and analyzed using CellQuest software.

Immunohistochemistry. Murine aortas were perfusion fixed, and segments containing large atherosclerotic lesions were embedded in paraffin. Blocks were sectioned, and sections were immunostained for oxidation-specific epitopes and macrophages (9). The presence of mouse Ig in lesions was detected by staining with antisera to mouse IgG and IgM (9), using biotinylated secondary Abs for detection. To assess the presence of EO/T15 murine autoantibodies in lesions, serial sections were stained with biotinylated AB1-2, an anti-T15 idiotype $\mathrm{Ab}(0.25 \mu \mathrm{g} / \mathrm{mL})$. As a control, adjacent sections were stained with the same protein concentration of an irrelevant biotinylated mouse IgG. Abs bound to the tissue were then detected with an
avidin-biotin-AP complex and phosphatase substrate. Endogenous tissue AP activity was blocked by 15 minutes of incubation with $5 \mathrm{mM}$ levamisole (Sigma Chemical Co.).

Genomic DNA PCR to determine the origin of EO bybridomas. PCR primers were designed to cover $\mathrm{V}_{\mathrm{H}}$ framework region 3 (FR3) (FR3S, 5'-ACT GCC ATT TAT TAC TGT GCA AGA G) and the downstream JH4 gene $3^{\prime}$ flanking region (JH4AS, 5'-AAC TCC ATA ACA AAG GTT AAA AAT AAA G). Independent PCR reactions were performed with $0.5 \mu \mathrm{M}$ of each primer and $2 \mu \mathrm{g}$ of genomic DNA template from each hybridoma in the optimized condition for 32 cycles. Genomic DNA extracted from mouse fibroblasts was used as a negative control. The PCR products were analyzed on $1 \%$ agarose gel and subcloned in a TA cloning vector (Invitrogen Corp.) for later sequence determination.

To further analyze the amplified fragments from these EO Ab-expressing clones, the PCR products were purified with QIAprep micro spinning columns (QIAGEN Inc.). The DNA was then digested with restriction endonuclease HindIII or BstNI, followed by $1 \%$ agarose gel electrophoresis to compare the fingerprints of the digested products.

\section{Results}

Genetic origins of EO Abs. Preliminary data documented that each of the EO B-cell hybridomas expressed an $\operatorname{IgM}-\kappa \mathrm{Ab}$ (data not shown). Using a strategy designed to enable the unbiased cloning of the vast majority of rearrangements among $\mathrm{V}_{\mathrm{H}}-\mu$ and $\mathrm{V}_{\mathrm{K}}$ genes (Table 1 ), RNA was extracted first from the EO6 cell line, the best characterized of the POVPC-specific EO Ab-expressing lines. In four of seven separate PCR reactions, each of which targeted different $\mathrm{V}_{\mathrm{H}} \mathrm{FR} 1$ sequences, amplimers of the expected size ( $380 \mathrm{bp})$ were obtained. After

\section{Table 1}

Primers designed to amplify $\mathrm{V}_{\mathrm{H}}$ and $\mathrm{V}_{\mathrm{K}}$ gene rearrangements of autoantibodies cloned from $A p o E^{-/-}$mice

\begin{tabular}{|c|c|c|}
\hline \multicolumn{3}{|c|}{$V_{H}$ FR1-specific sense primers } \\
\hline CLIII-FR1A & 7183 & 5'-GTGGAGTCTGGGGGAGGCTTA-3' \\
\hline CLIII-FR1B & $\mathrm{X} 24$ & 5'-GGAGGTGACCTGGTGCAGCCTGGA-3' \\
\hline CLIII-FRIC & J606 & 5'-GGAGGATGCTTGGTGCAACCTGGA-3' \\
\hline CLIII-FR1D & S107 & 5'-GGAGGAAGCTTGGTACAGCCTGGG-3' \\
\hline CLII-FRIA & Q52 & 5'-GGACCTGACCTGGTGCAGCCCTCA-3' \\
\hline CLI-FR1A & J558 & 5'-GGTGAAGCTTGGGGCTTCAGTGA-3' \\
\hline CLI-FR1B & Vgam3 & 5'-CAGATCCAGTTGGTGCAGTC-3' \\
\hline \multicolumn{2}{|c|}{$\mu$-specific antisense primer } & 5'-CATGGCCACCAGATTCTTA-3' \\
\hline \multicolumn{3}{|c|}{$V_{\kappa}$-degenerate sense primers } \\
\hline \multirow{2}{*}{\multicolumn{2}{|c|}{$\begin{array}{l}V_{\kappa}-\operatorname{deg} A \\
V_{\kappa}-\operatorname{deg} B\end{array}$}} & 5'-TCTCGAYATTTTGATTWHNCAGTC-3' \\
\hline & & 5'-TCTCGAYATTTTGATGACNCAR-3' \\
\hline \multicolumn{3}{|c|}{$\mathrm{J}_{\mathrm{K}}$-specific antisense primers } \\
\hline \multicolumn{2}{|l|}{$\mathrm{CJ}_{\kappa} 1,2$} & 5'-GGAAGATCTAGAGGAACCACCTTTKATTTCCAGYTTGGTCCC-3' \\
\hline \multicolumn{2}{|l|}{$\mathrm{CJ}_{\mathrm{K}} 4$} & 5'-GGAAGATCTAGAGGAACCACCTTTTATTTCCAACTTTGTCCC-3' \\
\hline \multicolumn{2}{|l|}{$\mathrm{CJ}_{\kappa} 5$} & 5'-GGAAGATCTAGAGGAACCACCTTTCAGCTCCAGCTTGGTCCC-3' \\
\hline
\end{tabular}

$V_{H}$ primers include seven sense primers specific to FR1 of repertoires, and an antisense primer specific to the $\mathrm{CH} 1$ domain of the $\lg \mu$ gene. $\mathrm{V}_{\mathrm{k}}$ primers include two degenerate $(\mathrm{deg})$ sense primers, which will be primed to $F R 1$ of most $V_{\kappa}$ genes, and three antisense primers specific to $J_{\kappa} 1,2, J_{\kappa} 4$, and $J_{\kappa} 5$ junction regions. 


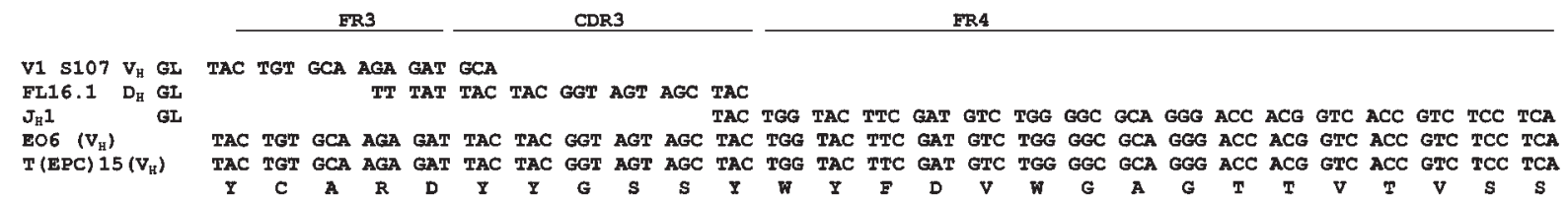

Figure1

The DNA sequences and VDJ splice sites of the somatic $V_{H}$ rearrangements expressed in the EO6 B-cell hybridoma and the $T(E P C) 15$ plasmacytoma, and their relationship to the most homologous germ-line $(G L) V_{H}, D_{H}$, and $J_{H}$ gene segments. Sequence analysis for the 372 encoding nucleotides of the EO6 $\mathrm{V}_{\mathrm{H}}$ rearrangement did not reveal a single nucleotide variation suggestive of somatic hypermutation. The canonical T15/EO6 VDJ rearrangement has been described as an archetype for primary homology-directed recombination, which more frequently occurs during B-lymphogenesis in the fetal liver. CDR: complementarity-determining region.

independent cloning and DNA sequence determinations, each of the cloned amplimers was found to represent the same unique in-frame $\mathrm{V}_{\mathrm{H}}-\mu$ gene rearrangement, derived from the S107 family. This gene rearrangement was $100 \%$ identical to a previously reported nonmutated, functional, canonical $V_{H S 1071^{-}}$ $\mathrm{D}_{\mathrm{FL} 16.1}-\mathrm{J}_{\mathrm{H}} 1$ rearrangement that is also expressed in the $\mathrm{T}(\mathrm{EPC}) 15$ IgA-K plasmacytoma (10) (Figure 1). Amplimers of the expected size ( $350 \mathrm{bp})$ were obtained in two of six separate $V_{\mathrm{K}}$ gene amplification reactions of cDNA from EO6 cells (Table 1). Sequence determination of plasmids containing these independently cloned inserts yielded the same in-frame nonmutated, canonical $\mathrm{V}_{\mathrm{K}} 22-\mathrm{J}_{\mathrm{K}} 5$ rearrangement that was also previously reported for the T(EPC) 15 plasmacytoma.

To independently confirm the genetic origin of the $\mathrm{V}_{\mathrm{H}}$ gene expressed by the EO6 cell line, separate RTPCR reactions were performed using primers specific to unique upstream leader-specific sequences that can discriminate between the V1 gene and the V11/13 functional genes of the S107 family. Amplification was obtained only for the V1-specific primer, and subsequent sequence analysis of the cloned product confirmed the exact nonmutated $\mathrm{Ab}$ gene sequence identified in the earlier $\mathrm{V}_{\mathrm{H}}$ gene cloning studies.

Equivalent studies were also performed with cDNA from three other OxLDL-reactive POVPC-specific cell lines. As with EO6, DNA sequence analysis of the $V_{H}$ and $\mathrm{V}_{\mathrm{L}}$ genes of the EO3, EO4, and EO7 cell lines indicated that they were each $100 \%$ homologous to the classical T15 Ab. Figure 2 shows the deduced amino acid sequences for these $\mathrm{Abs}$; the $\mathrm{Ab}$ gene usage for each of these EO cell lines and their binding reactivities (6) are compiled in Table 2.

$E O$ autoantibodies are recognized by $T 15$-specific anti-idiotypes. To characterize the $\mathrm{V}_{\mathrm{H}}$ and $\mathrm{V}_{\mathrm{L}}$ regions expressed in the different $\mathrm{EO} \mathrm{Abs}$ and determine their relationship to the classic $\mathrm{T} 15 \mathrm{Ab}$, we tested the recognition of these Abs by three well-characterized T15-specific antiidiotypic Abs. These were T139.2, directed against the unique $\mathrm{T} 15$-specific $\mathrm{V}_{\mathrm{K}} 22-\mathrm{J}_{\mathrm{K}} 5$ light chain (11); Tc54.6, directed against the unique T15-specific $\mathrm{V}_{\mathrm{HS} 107.1}$ usage $(11,12)$; and AB1-2, directed against a conformational determinant requiring both the heavy- and light-chain CDR3 regions of T15 (13). As shown in Table 2, each of the POVPC-specific, anti-OxLDL Abs for which the $V_{H}$ and $\mathrm{V}_{\mathrm{L}}$ region sequences were determined (EO3, EO4, EO6, and EO7) also expressed the T15 idiotype (i.e., they are recognized by each of three anti-idiotypic Abs). In addition, three other POVPC-specific autoantibodies, EO1, EO2, and EO5 (6), were also found to express the T15 idiotype (data not shown). In contrast, EO12 and EO14, which bind MDA-LDL (3), did not express any of the T15 markers.

Binding of EO6/T15 to OxLDL and PC. T15 is a classic $\mathrm{Ab}$ to PC, the "headgroup" moiety [-PO $\left.\mathrm{PO}_{4}-\left(\mathrm{CH}_{2}\right)_{2}-\mathrm{N}-\left(\mathrm{CH}_{3}\right)_{3}\right]$ of many phospholipids, including PAPC. Because the genetic and idiotypic analyses indicated that these EO autoantibodies to OxLDL used $\mathrm{V}_{\mathrm{H}}$ and $\mathrm{V}_{\mathrm{L}}$ regions identical to those of $\mathrm{T} 15$, we systematically compared the antigen-binding properties of $\mathrm{T} 15$ (an IgA) with a representative EO Ab, EO6 (an IgM). As expected, EO6 bound

Table 2

Summary of gene usage and immunological properties of T15 and autoantibodies from apoE-deficient mice

\begin{tabular}{|c|c|c|c|c|c|c|c|c|c|c|}
\hline $\begin{array}{l}\text { Clone } \\
\text { name }\end{array}$ & $V_{H}$ usage & $V_{L}$ usage & $\begin{array}{c}\text { AB1-2 } \\
\text { (S107.1/K22) } \\
\text { idiotype }\end{array}$ & $\begin{array}{c}\text { T139.2 } \\
\text { (к22) } \\
\text { idiotype }\end{array}$ & $\begin{array}{l}\text { Tc54.6 } \\
\text { (S107.1) } \\
\text { idiotype }\end{array}$ & $\begin{array}{l}\text { Binding to } \\
\text { Cu-OxLDL }\end{array}$ & $\begin{array}{l}\text { Binding to } \\
\text { POVPC-BSA }\end{array}$ & $\begin{array}{l}\text { Binding to } \\
\text { MDA-LDL }\end{array}$ & $\begin{array}{l}\text { Binding to } \\
\text { PC-KLH }\end{array}$ & $\begin{array}{l}\text { Binding to } \\
\text { C-PS }\end{array}$ \\
\hline EO3 & S107.1 & $\kappa 22$ & ++++ & ++++ & ++++ & ++++ & ++++ & - & ++++ & ++++ \\
\hline EO4 & S107.1 & $\kappa 22$ & ++++ & ++++ & ++++ & ++++ & ++++ & - & ++++ & ++++ \\
\hline EO6 & S107.1 & $\kappa 22$ & ++++ & ++++ & ++++ & ++++ & ++++ & - & ++++ & ++++ \\
\hline EO7 & S107.1 & $\kappa 22$ & ++++ & ++++ & ++++ & ++++ & ++++ & - & ++++ & ++++ \\
\hline EO12 & ND & ND & - & - & - & - & - & ++++ & - & - \\
\hline EO14 & ND & ND & - & - & - & - & - & ++++ & - & - \\
\hline T15 & S107.1 & $\kappa 22$ & ++++ & ++++ & ++++ & ++++ & ++++ & - & ++++ & ++++ \\
\hline
\end{tabular}

Clones secreting EO Abs were sequenced and analyzed for their germline gene usage, and compared with the T15 Ab. Their recognition by anti-T15 idiotypic Abs (AB1-2, T139.2, and Tc54.6) and their binding to oxidation epitopes (Cu-OxLDL, MDA-LDL, and POVPC-BSA) and classical PC epitopes (PC-KLH, C-PS) are compared. Note the identical patterns of EO Abs and T15. ND, not determined. 


\section{Figure 2}

Reduced amino acid sequence alignment of EO autoantibodies with the classic anti-PC Ab T15. (a) Variable region of Ig light-chain sequences of EO autoantibodies are aligned against $\mathrm{V}_{\mathrm{K}} 22 / \mathrm{J}_{\kappa} 5$ gene rearrangement. (b) Variable regions of heavy-chain sequences of EO autoantibodies are aligned against $S 107.1 / D_{\mathrm{FL} 16.1} / \mathrm{J}_{\mathrm{H}} 1$ rearrangement. Both $V_{H}$ and $V_{L}$ show $100 \%$ homology to the germline genes of T15.

to $\mathrm{Cu}-\mathrm{OxLDL}$ and POVPC-BSA, but not to native LDL or MDA-LDL (6) (Figure 3a). As reported previously, T15 bound to its known antigen, $\mathrm{PC}$, derivatized onto the carrier proteins BSA and KLH (data not shown) (14). Like EO6, T15 also bound to Cu-OxLDL and POVPC$\mathrm{BSA}$, but not to native LDL or MDA-LDL (Figure $3 \mathrm{~b}$ ). In addition, with reactivity similar to that of T15, EO6 bound to the PC conjugates PC-BSA and PC-KLH. Neither EO6 nor T15 bound to the native, unoxidized phospholipid PAPC (data not shown).

To further investigate the fine binding specificity of EO6 to PC, a competition immunoassay was performed. Figure 4 shows that the binding of $\mathrm{EO} 6$ to $\mathrm{Cu}-$ OxLDL was totally inhibited by soluble PC (PC-Cl) and by PC-KLH. In analogous experiments, the binding of $\mathrm{T} 15$ to $\mathrm{Cu}-\mathrm{OxLDL}$ was also inhibited by $\mathrm{PC}-\mathrm{Cl}$ in a dose-dependent manner (data not shown). In control experiments, the binding of another oxidation-specific IgM monoclonal autoantibody, EO14, to its antigen, MDA-LDL, was unaffected by PC-Cl (data not shown).

During the oxidation of LDL, a large number of reactive lipid peroxidation products are generated that can serve as epitopes for $\mathrm{Ab}$ recognition. Therefore, we directly tested the ability of T15 to compete with biotinylated EO6 for binding to OxLDL. The inset in Figure 4 demonstrates that $\mathrm{T} 15$ competed effectively, whereas a control murine IgA did not. This suggests that these two mAb's are directed against an immunologically closely related (if not identical) epitope on OxLDL. In addition, we demonstrated that the binding of EO6 to PC-KLH was inhibited by both PC-KLH and Cu-OxLDL in a dosedependent manner (data not shown). In addition, the a

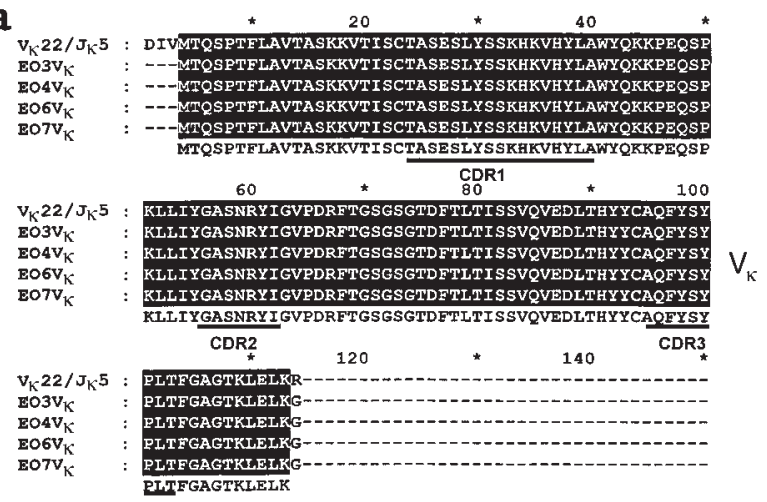

b

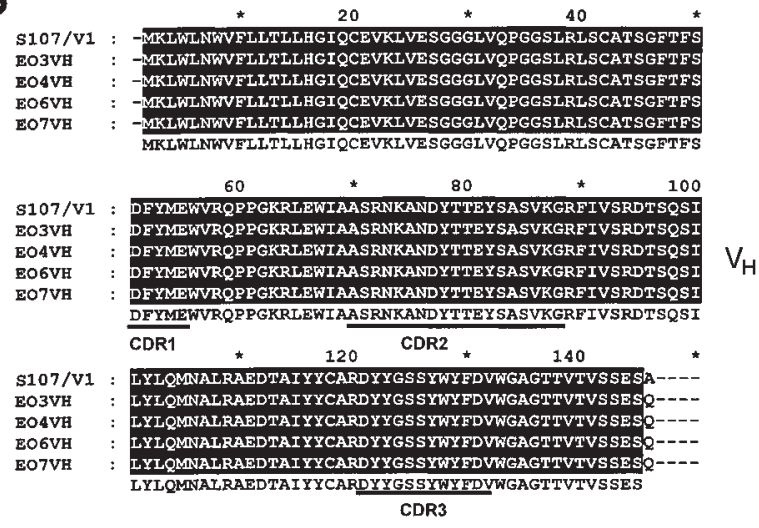

demonstration that the anti-idiotype T139.2 competed very effectively with EO6 for binding to $\mathrm{PC}-\mathrm{KLH}$ provided further confirmation that the binding of EO6 to PC uses the same antigen-binding site used by the classic $\mathrm{T} 15 \mathrm{Ab}$ (data not shown).

EO6 and T15 bind to bacterial C-PS. PC is the immunodominant determinant in the teichoic acid-containing C-PS of Streptococcus pneumoniae (15), and both EO6 and T15 bound to C-PS, whereas EO14 (an MDA-LDL-specific $\mathrm{Ab}$ ) did not (Figure 5a). Furthermore, the binding of both EO6 and T15 to OxLDL was dose-dependently inhibited by the addition of C-PS (Figure $5 \mathrm{~b}$ ). These data indicate that both EO6 and T15 recognize a common

\section{Figure 3}

Binding of EO6 and T15 to oxidationspecific epitopes of LDL and PC. The indicated antigens were plated on microtiter wells at the indicated concentrations overnight at $4^{\circ} \mathrm{C}$. EO6 (a) or T15 (b) was added at $5 \mu \mathrm{g} / \mathrm{mL}$ followed by the AP-conjugated goat antimouse IgM (for EO6) or IgA (for T15) secondary Abs. The amount of bound Abs was expressed as RLU/100 ms. Nat LDL, native LDL. a

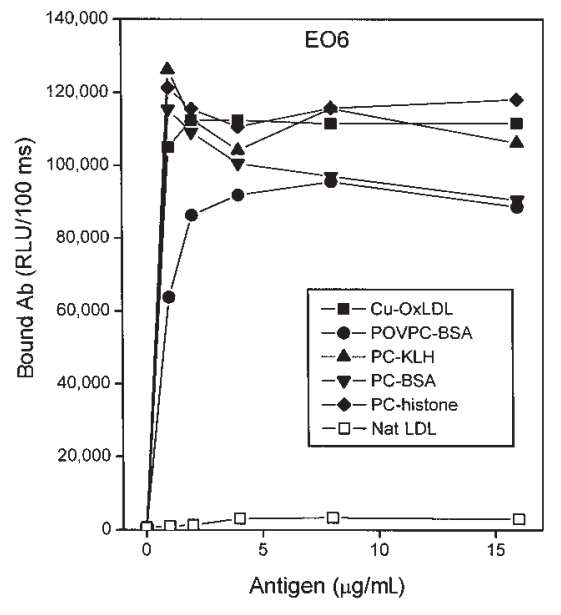

b

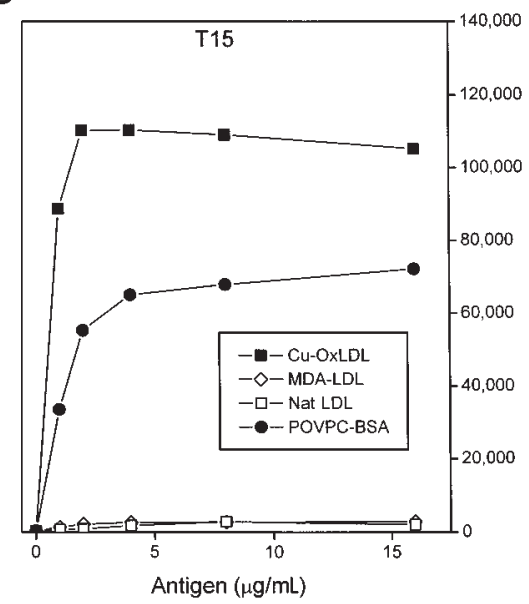




\section{Figure 4}

Inhibition of EO6 binding to $\mathrm{Cu}-\mathrm{OxLDL}$ by $\mathrm{PC}$, as sodium salt (PC$\mathrm{Cl}$ ) or PC-KLH conjugate. Cu-OxLDL $(10 \mu \mathrm{g} / \mathrm{mL})$ was plated on microtiter wells overnight at $4{ }^{\circ} \mathrm{C}$. EO6 $(10 \mu \mathrm{g} / \mathrm{mL})$ was added to wells in the absence or presence of the indicated concentrations of competitors, and the amount of bound EO6 was detected by AP-conjugated goat anti-mouse IgM. The amount of bound EO6 was expressed as the percent of EO6 binding to Cu-OxLDL in the absence of competitor. Inset: Inhibition of EO6 binding to $\mathrm{Cu}$ OxLDL by both EO6 and T15. Cu-OxLDL $(10 \mu \mathrm{g} / \mathrm{mL})$ was coated on microtiter wells overnight at $4^{\circ} \mathrm{C}$. Biotinylated EO6 $(10 \mu \mathrm{g} / \mathrm{mL})$ was added to microtiter wells in the absence or presence of the indicated concentrations of competitors. The amount of biotinylated EO6 bound to $\mathrm{Cu}-\mathrm{OxLDL}$ was then detected by AP-conjugated NeutrAvidin ${ }^{\circledR}$. The amount of biotinylated EO6 bound to the antigen in the absence of competitor was expressed as a percentage of the control. Nonspecific mouse IgA was used as isotype control for T15.

epitope present on OxLDL as well as on C-PS.

T15 Abs recognize apoptotic but not normal endothelial cells. We have previously shown that EO6 bound to apoptotic cells and inhibited their phagocytosis by macrophages (7). To test whether this was a common feature of all T15 Abs regardless of isotype, we examined the capacity of the classic IgA T15 to bind to apoptotic PAECs by flow cytometric analysis. In these studies, serum deprivation was used to induce apoptosis in PAECs. As illustrated in Figure 6, T15 preferentially bound to PAECs that were in advanced stages of apoptosis (Figure 6c), but not to normal cells or those in very early stages of apoptosis (Figure 6b).

Abs with the T15 idiotype are present in atherosclerotic lesions. We previously demonstrated that EO6 immunostains OxLDL-associated determinants in atherosclerotic lesions from rabbits and humans (3). We have also shown that autoantibodies to OxLDL are present in lesions, in part complexed to OxLDL (16). To determine whether some of the anti-OxLDL Abs deposited in the atherosclerotic lesions of mice consist of the EO6 and/or T15 idiotype, we immunostained advanced lesions from cholesterol-fed $L D L R^{-/-}$ mice for the presence of Abs expressing the T15/EO6 idiotype. Figure 7a demonstrates the marked deposi-

\section{Figure 5}

Binding of EO6 and T15 to pneumococcal C-PS. (a) C-PS was plated on microtiter wells at the indicated concentrations overnight at $4^{\circ} \mathrm{C}$. Each $\mathrm{Ab}(5 \mu \mathrm{g} / \mathrm{mL})$ was added to the plate and detected by APconjugated secondary Abs (goat anti-mouse IgM for EO6 and EO14, goat anti-mouse IgA for T15). The amount of bound $A b$ was expressed as RLU/100 ms. (b) Cu-OxLDL or MDA-LDL $(10 \mu \mathrm{g} / \mathrm{mL})$ was plated as antigen, and the indicated $A b$ was added to microtiter wells in the absence or presence of the indicated concentrations of C-PS. Bound Ab was detected by AP-conjugated secondary Abs (goat anti-mouse IgM for EO6 and EO14, goat anti-mouse IgA for T15), and was expressed as percent control of $A b$ binding to its antigen without competitor.

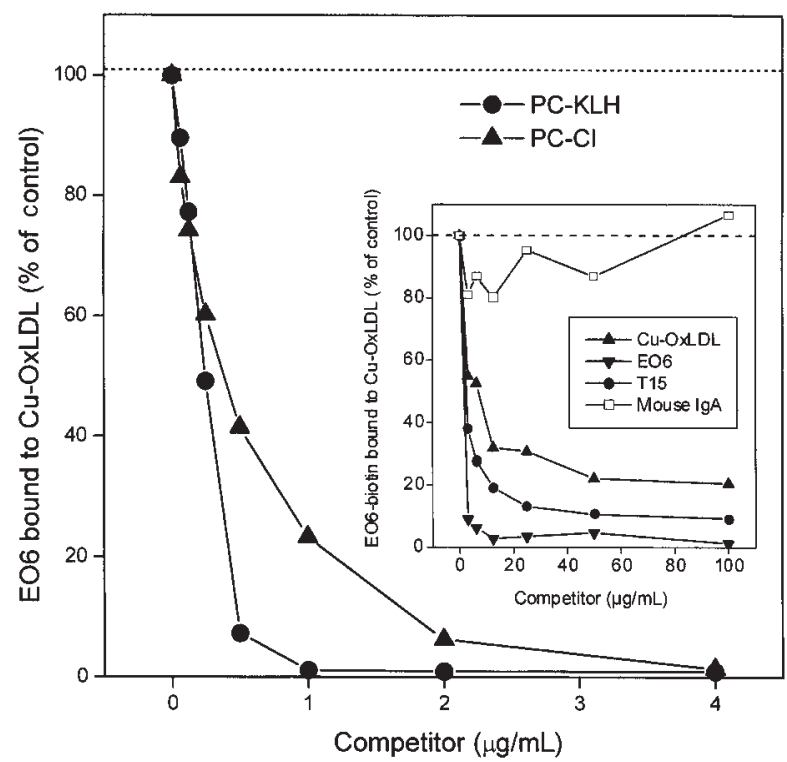

tion of Ig in murine lesions, as reported previously (9). In Figure $7 b$, an adjacent section was stained with the biotinylated AB1-2 anti-T15 idiotype, which yielded the same pattern of staining depicted for total Ig deposition. An adjacent section stained with an equivalent concentration of a biotinylated isotype control did not reveal staining (Figure 7c). In aortic sections from other diseased mice, specific staining with this anti-idiotypic reagent was also demonstrated, although the extent of T15 immunostaining varied in different atherosclerotic lesions (not shown). Because staining with $\mathrm{AB} 1-2$ recognizes a T15-specific idiotope that is independent of the associated isotype or isotypes (e.g., IgA, IgM, or IgG), these studies cannot distinguish the isotypes of the deposited T15 idiotype-bearing Ig's. Nevertheless, these studies document that T15 and EO6 Abs are specifically deposited in lesions of murine atherosclerosis.

$O x L D L$ - and POVPC-reactive EO cell lines display bighly restricted clonal heterogeneity. To evaluate their clonal heterogeneity, a PCR-based strategy was developed to characterize the genomic $V_{H}$ rearrangements in OxLDLand POVPC-reactive EO cell lines, using conditions wherein nonrearranged genomic templates do not yield an amplimer product (see Methods). In previous stud- 


\section{Figure 6}

T15 IgA recognizes apoptotic cells. FACS ${ }^{\circledR}$ analysis of T15 Ab and control IgA (C-IgA; nonspecific monoclonal) binding to apoptotic PAECs. (a) Apoptosis-induced PAECs were gated into two populations according to PI intensity and FSC as described (7). Region 2 (R2): normal cells and cells in very early apoptosis. Region 1 (R1): cells with dim and bright PI staining (apoptotic). (b) Binding of control IgA and T15 to R2 cells. (c) Binding of control IgA and T15 to R1 cells.

ies, we demonstrated that EO6 cells expressed transcripts for the productive $\mathrm{T} 15 \mathrm{~V}_{\mathrm{H}}$ gene (S107.1- $\mathrm{D}_{\mathrm{FL} 16.1}$ $\mathrm{J}_{\mathrm{H}} 1$ ) and also a nonproductive, frame-shifted S107.13$\mathrm{D}_{\mathrm{SP} 2.2}-\mathrm{J}_{\mathrm{H}} 3$ rearrangement. Confirming these findings, amplification of the genomic DNA of EO6 cells yielded two bands, with a larger, 1,500-bp product shown by DNA sequence analysis to represent the productive T15 $\mathrm{V}_{\mathrm{H}}$ gene and flanking sequence, and a smaller 800-bp product that represents the nonproductive S107 rearrangement on another allele. Significantly, the other OxLDL- or POVPC-specific cell lines, EO3, EO4, and $\mathrm{EO}$, also yielded only bands of these exact same sizes, which were shown to be identical by sequence analysis and HindIII and BstNI fingerprint patterns (data not shown). Notably, amplification of the genomic DNA of the fusion partner used to create these cell lines yielded only the smaller (800-bp) band, indicating that in the EO cell lines this band was a remnant of the fusion event, and not a marker of the rescued B cells. These data provide compelling evidence that each of these OxLDL- or POVPC-reactive EO hybridomas are genetically identical, likely arising from the same clonal set of B lymphocytes that are specific for an oxidation-associated neodeterminant.

\section{Discussion}

Natural autoantibodies are predominately IgMs that constitute a significant fraction of serum Ig's at birth and may increase with age. Many of these Abs are encoded by nonmutated germline $V_{H}$ or $V_{L}$ genes (17), and they react with a variety of self-determinants, such as carbohydrates and glycolipids, and often cross-react with bacterial or oncofetal antigens to provide a fundamental tier of the adaptive immune system. The developmentally regulated processes responsible for the spontaneous production of natural autoantibodies are not clearly understood. We now report that a set of antibodies expressed by a disease-associated dominant B-cell clonal set, directed against oxidation-specific epitopes present on OxLDL and apoptotic cells, is identical to classic T15 natural autoantibodies that dominate anti-PC responses and provide optimal protection from bacterial infection from virulent $S$. pneumoniae (18).

We documented previously that $\mathrm{Ab}$ titers to oxidationspecific epitopes of OxLDL, including those of the IgM isotype, increase in parallel with the development of atherosclerosis in mice (9). Examination of the immune response occurring in atherosclerotic $\mathrm{ApoE}^{-/-}$mice demonstrated that at least $32 \%$ of 1,536 viable, Ig-secret-
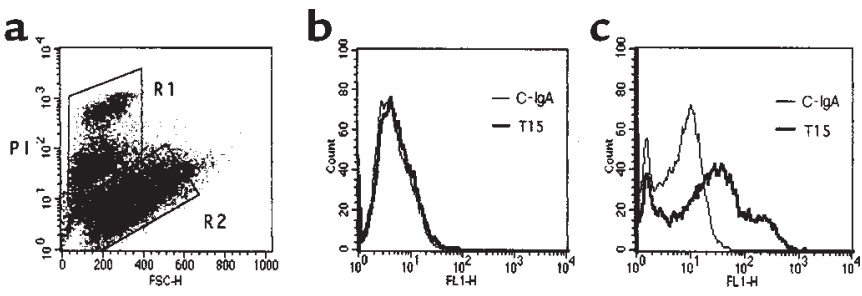

ing hybridomas obtained from a single splenic B-cell fusion event secreted Abs that bound to epitopes of OxLDL (3). Indeed, the weights of the spleens of $\mathrm{ApoE}^{-/-}$ mice were $40 \%$ greater than those of controls, which is consistent with the extraordinarily exuberant immune response to the various oxidation-specific epitopes that occurs with the development of advanced atherosclerosis and enhanced lipid peroxidation (3). Gene sequence analysis and anti-idiotypic studies revealed that all seven hybridomas secreting POVPC-specific IgMs expressed the same nonmutated germline $V_{H}$ and $V_{L}$ genes that are expressed by the classic anti-PC B-cell clone, T(EPC) 15 . Adapting an approach previously described by Shan et al. (19), we analyzed the genomic $V_{H}$ rearrangements in the POVPC-specific hybridomas, which suggested that these cell lines all derived in vivo from an expansion of the same clonal set of antigen-stimulated B cells, and do not represent independent clonal outgrowths or reflect nonspecific polyclonal or mitogenic stimulation. Although we cannot absolutely rule out the possibility that these cell lines reflect the ex vivo expansion of a single lymphocyte clone, this seems highly unlikely given the large number of cell lines isolated. These findings are therefore most consistent with in vivo selection by apparently immunodominant, oxidation-associated phospholipid epitopes. Thus, our data are most consistent with the hypothesis that during progression of atherosclerosis there is an in vivo expansion of the T15/EO6 clonal set in the $A p o E^{-/-}$mice; preliminary studies support this hypothesis (G. Silverman et al., unpublished observation). The finding of marked deposition of T15 idiotype Abs in atherosclerotic lesions (Figure 7) also strongly supports this interpretation. Also supporting this hypothesis, Itabe and colleagues have reported that after immunization with human atheromatous material, they isolated a B-cell line producing an $\mathrm{Ab}$ that appears to be nearly identical to our $\operatorname{EO} 6 \mathrm{Ab}(6,20)$.

The discovery that the $\mathrm{V}_{\mathrm{H}}$ and $\mathrm{V}_{\mathrm{L}}$ gene rearrangements of the anti-POVPC Abs are identical to a group of classic anti-PC Abs that have been extensively studied for the past 30 years is of considerable interest. The biologic importance of the T(EPC) 15 clone, an IgAexpressing plasmacytoma, derives from the fact that $\mathrm{B}$ cell clones with the T15 Ab-defining genes are represented at high frequency in many inbred mouse strains, without prior immune exposure. In fact, the majority of Abs to PC, the immunodominant moiety in C-PS of pneumococci and a common constituent of other pathogens (15), bear the T15-specific idiotypic mark- 

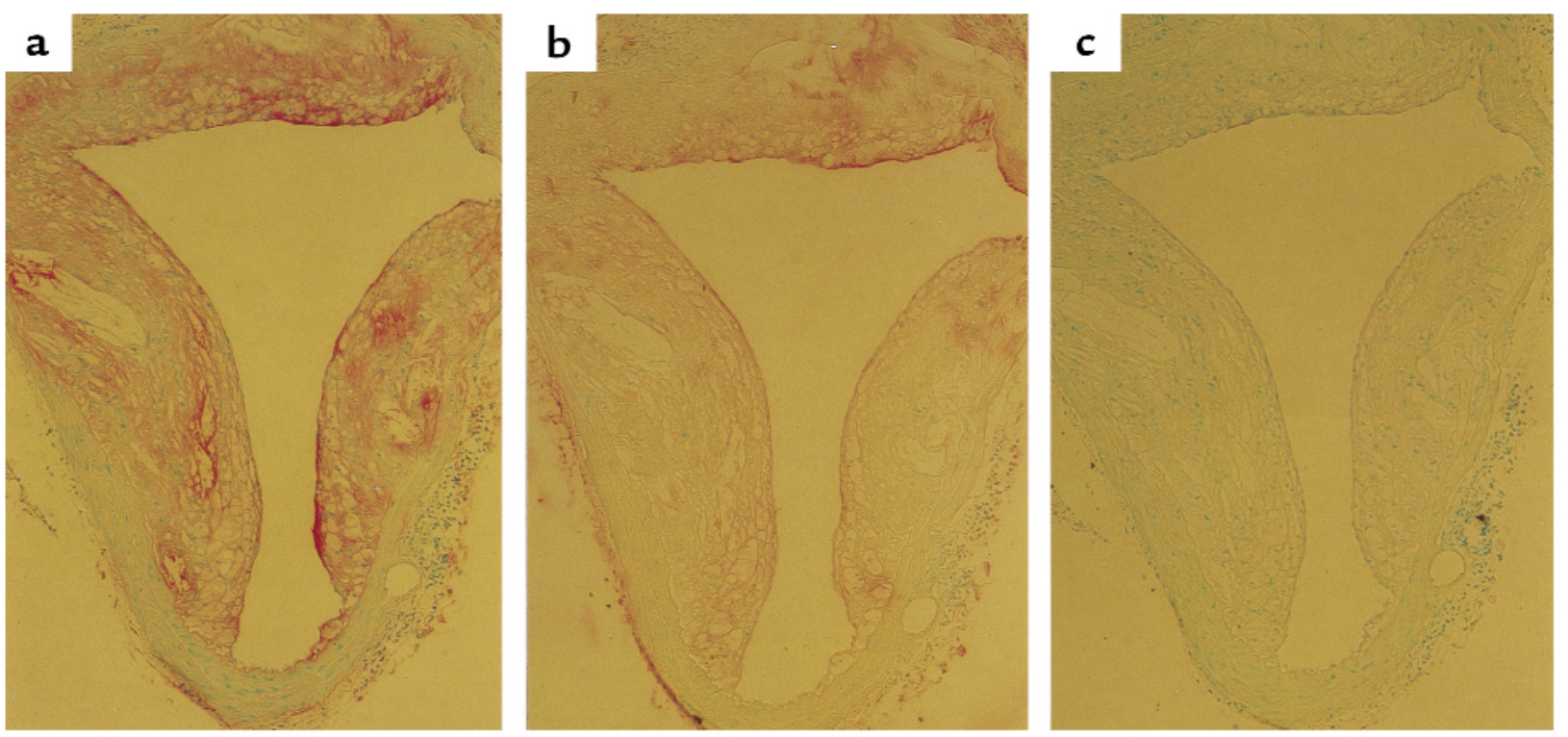

\section{Figure 7}

Presence ofT15 idiotypic Abs in atherosclerotic lesions. Atherosclerotic segments of aorta of $L D L R^{-/-}$mice were prepared and stained as described in Methods. Epitopes recognized are indicated by a red color; the nuclei are counterstained with methyl green. (a) Section immunostained with a combined anti-mouse IgG/IgM antisera, indicating the presence of Ig in lesion. (b) An adjacent section immunostained with biotinylated AB1-2 (anti-T15 idiotype). A similar pattern demonstrates that some Ig deposited in the lesion represents Abs of the T15 idiotype. (c) An adjacent section stained with biotinylated nonimmune $\lg G$ was free of specific staining.

ers. Moreover, beginning from about day 6 of life, T15 Abs dominate in vivo responses to PC-containing immunogens (21), and they are the most protective of all anti-PC Abs to systemic infection with pneumococci (18). However, despite their importance and the large amount of investigative interest they have generated, the mechanism or mechanisms responsible for their in vivo dominance have remained a mystery, as they are highly represented even in germ-free mice (22). Therefore, the current findings are important because they identify previously unsuspected relevant "neo-self" ligands for the T15 clonal set that appear to contribute to the selection and maintenance of an important component of the host's preformed immune defense from microbial attack.

Adoptive transfer studies have rigorously documented that T15 B cells exist predominantly (or solely) as part of the $\mathrm{CD}^{+} \mathrm{B}-1$ pool (23). Although B- 1 cells normally constitute only a minor fraction of peripheral lymphoid tissue (such as spleen), they represent a major fraction of B cells found in the peritoneal cavity of mice, and are the source of most natural IgMs that spontaneously arise in naive hosts without specific immunization (24). Hence, it is speculated that these primordial $\mathrm{B}$ cells represent evolutionarily selected $\mathrm{Ab}$ genes and binding specificities that dominate the repertoire expressed during early ontogeny, in part to serve housekeeping functions of removing everyday "self" debris required for maintaining homeostasis. Our findings that classical T15 Abs bind to oxidationspecific neodeterminants on both OxLDL and apoptotic cells may therefore represent the first demonstration of a defined autoreactivity by the T15 B-cell set.
These novel observations demonstrate the endogenous antigens that could be responsible for positive selection, resulting in the progressive in vivo dominance of the T15 anti-PC B-cell set.

Negative selection has long been thought to be the dominant mode of regulation of B-cell development in health, though the presence of natural autoantibodies is in seeming conflict with this theory (25). Although a positive role for self-reactivity in B-cell development has been previously considered, several recent reports have now provided firm evidence that mature B cells can be affected by receptor-mediated positive selection based on their autoreactivity. Hayakawa and colleagues (26) generated a murine transgenic model in which coexpression of an unmutated $\mathrm{V}_{\mathrm{H}}$ rearrangement encoding for self-specificity for Thy- 1 glycoprotein (CD90), along with its natural self-ligand on T cells, resulted in the positive selection of the Tg B-1 cell population and enhanced serum autoantibody levels. Their important findings modify the view, based on most earlier Tg-Ig systems, that autoreactive binding of highaffinity or high-avidity determinants, constitutively expressed throughout immune maturation, results in B-cell clonal anergy or deletion. Several other recent reports also support the notion of positive antigen selection of B-1 cells $(27,28)$. Although these reported systems have similarities to the T15 B-1 subset, our findings are novel in that they strongly implicate B-1 cells in immune response to oxidation-specific epitopes that are linked to a pathologic state, atherosclerosis.

The binding of T15/EO6 Abs is highly specific for oxidized PAPC, and they do not bind to unoxidized PAPC, a prominent phospholipid of LDL and cells. Because 
the PC headgroup is present in many phospholipids, including PAPC, and the T15/EO6 Abs do not bind native PAPC, it must be that oxidation alters the configuration or accessibility of PC so that it is able to fit into the antigen-binding site. These findings are potentially important in light of the extensive structural observations that have been made for PC-binding Abs. Crystallography studies of the hapten-binding site of a Group I anti-PC Ab similar to $\mathrm{T} 15$ revealed that PC occupied only a small part of the antigen-binding pocket, with the choline moiety in the interior (29). We speculate that changes to other parts of the phosphatidylcholine molecule caused by oxidation, or by formation of covalent adducts between the core aldehyde of the oxidized $\operatorname{sn} 2$ fatty acid and a lysine group of a protein (6), can make the PC headgroup in phosphatidylcholine accessible for T15 and EO6 binding. Alternatively (or additionally), it is possible that both the sn2 oxovaleroyl group and the sn3 PC fit into the antigen-binding site simultaneously, with the $s n 1$ hydrophobic saturated fatty acid remaining outside the antigen-binding pocket. In either case, the "true antigen" appears to be an oxidized phospholipid, such as POVPC, that is predicted to have a higher binding affinity. This would suggest that other oxidized phospholipids containing the PC headgroup might also fit into the same pocket. Similarly, we speculate that PC and perhaps other similar oxidized phospholipid moieties become expressed on OxLDL and the surface of apoptotic cells as they undergo oxidative changes known to accompany apoptosis.

The EO Abs bearing the T15 idiotype, such as EO6, have the capacity to block the uptake of OxLDL by macrophages (6). Furthermore, the epitopes recognized by EO6 are present on minimally oxidized LDL found in the circulation (3). Thus, we speculate that these Abs could affect atherosclerosis both by preventing foam-cell formation and by leading to enhanced removal from plasma of such minimally oxidized LDL, preventing their deposition in the artery wall. Similarly, these Abs might also influence the disposition of apoptotic cells (7). The observation that the same Abs also bind to C-PS, by its PC epitopes, raises the fascinating possibility that pneumococcal (or other) infections, or conceivably even vaccination, could increase the titers of T15 idiotypic Abs; this in turn could impact atherogenesis, as noted above. Obviously, extensive in vivo studies will be needed to test these speculative observations.

In this discussion we have referred to oxidation-specific epitopes generated on OxLDL and apoptotic cells as "neodeterminants," and to the T15 and EO6 Abs that recognize them as "autoantibodies." It is a matter of conjecture whether these are truly self antigens and autoantibodies in the classical sense. Indeed, we hypothesize that many disease-associated autoantibodies are in fact directed against subtle modifications of self proteins or lipids that have not been previously recognized. For example, we demonstrated previously that some of the so-called "anti-phospholipid autoantibodies," found in patients with anti-phospholipid Ab syndrome, in fact bound to oxidized phospholipids but not to native phospholipids $(30,31)$. Based on the current findings, we speculate that other B-1 cell clones that secrete natural housekeeping autoantibodies will also be found to be specific for a variety of neo-self epitopes generated by common modifications, such as other oxidation-dependent epitopes, and enzymatic and nonenzymatic glycation.

These data suggest a new immunologic paradigm whereby immune recognition (both humoral and cellmediated) of oxidation-specific epitopes contributes to physiologic homeostasis of apoptotic and necrotic cells, as well as senescent cells (such as erythrocytes) and lipoproteins (and similar lipid-rich membranes) that have undergone oxidative changes. This process is integral to normal physiology, because we exist in an aerobic environment. Indeed, we have recently demonstrated the presence of OxLDL even in fetal arteries (32). Furthermore, apoptosis is a normal developmental and homeostatic prerequisite. Hence, we hypothesize that neo-self oxidized phospholipids have served as an evolutionary pressure contributing to the selection of this primordial B-cell set. Under conditions of enhanced oxidative stress, as occurs in the $A p o E^{-/-}$mouse with hypercholesterolemia and extensive atherosclerosis, we speculate that there is an in vivo expansion of the T15 B-1 cell set. Our data support the hypothesis that natural selection has contributed to the conservation and recurrent expression of the T15 B-cell clone during early immune development, presumably both for its housekeeping roles in health and for the advantages it provides for natural immunity from certain common infectious agents. The specific role or roles of these T15 and EO Abs in the development (or amelioration) of atherosclerosis require further elucidation.

\section{Acknowledgments}

The authors acknowledge the advice and helpful discussion of Daniel Steinberg, Edward Dennis, Peter Friedman, and Alan Stall. These studies were supported by postdoctoral research fellowships from the American Heart Association Western Affiliate (P.X. Shaw and S. Hörkkö), by a startup grant from the Stein Institute of Research on Aging (P.X. Shaw), by National Heart, Lung, and Blood Institute grants HL56989 (Specialized Center of Research in Molecular Medicine and Atherosclerosis; J.L. Witztum, principal investigator) and HL57505 (J.L. Witztum), by National Institutes of Health grant RO1 AI-40305 (G.J. Silverman), and by the Multipurpose Arthritis and Musculoskeleton Disease Center Molecular Biology Core (grant AR40770). We thank H.K. Kim, Head of the Division of Nephrology, Korea University Hospital, for generous support of M.-K. Chang.

\footnotetext{
1. Steinberg, D., and Witztum, J.L. 1999. Lipoproteins, lipoprotein oxidation, and atherogenesis. In Molecular basis of cardiovascular disease. K.R. Chien, editor. W.B. Saunders Co. Philadelphia, Pennsylvania, USA. $458-475$.

2. Palinski, W., et al. 1994. ApoE-deficient mice are a model of lipoprotein oxidation in atherogenesis. Demonstration of oxidation-specific epitopes in lesions and high titers of autoantibodies to malondialdehyde-lysine in serum. Arterioscler. Thromb. 14:605-616.
} 
3. Palinski, W., et al. 1996. Cloning of monoclonal autoantibodies to epitopes of oxidized lipoproteins from apolipoprotein E-deficient mice. Demonstration of epitopes of oxidized low density lipoprotein in human plasma. J. Clin. Invest. 98:800-814.

4. Palinski, W., Miller, E., and Witztum, J.L. 1995. Immunization of low density lipoprotein (LDL) receptor-deficient rabbits with homologous malondialdehyde-modified LDL reduces atherogenesis. Proc. Natl. Acad. Sci. USA. 92:821-825.

5. Freigang, S., Hörkkö, S., Miller, E., Witztum, J.L., and Palinski, W. 1998 Immunization of LDL receptor-deficient mice with homologous malondialdehyde-modified and native LDL reduces progression of atherosclerosis by mechanisms other than induction of high titers of antibodies to oxidative neoepitopes. Arterioscler. Thromb. Vasc. Biol. 18:1972-1982.

6. Hörkkö, S., et al. 1999. Monoclonal autoantibodies specific for oxidized phospholipids or oxidized phospholipid-protein adducts inhibit macrophage uptake of oxidized low-density lipoproteins. J. Clin. Invest. 103:117-128.

7. Chang, M., et al. 1999. Monoclonal antibodies against oxidized lowdensity lipoprotein bind to apoptotic cells and inhibit their phagocytosis by elicited macrophages: evidence that oxidation-specific epitopes mediate macrophage recognition. Proc. Natl. Acad. Sci. USA. 96:6353-6358

8. Silverman, G.J., et al. 1998. The dual phases of the response to neonatal exposure to a VH family-restricted staphylococcal B cell superantigen. J. Immunol. 161:5720-5732.

9. Palinski, W., Tangirala, R.K., Miller, E., Young, S.G., and Witztum, J.L. 1995. Increased autoantibody titers against epitopes of oxidized LDL in LDL receptor-deficient mice with increased atherosclerosis. Arterioscler. Thromb. Vasc. Biol. 15:1569-1576.

10. Gearhart, P.J., Johnson, N.D., Douglas, R., and Hood, L. 1981. IgG antibodies to phosphorylcholine exhibit more diversity than their IgM counterparts. Nature. 291:29-34.

11. Kenny, J.J., et al. 1992. Antigen binding and idiotype analysis of antibodies obtained after electroporation of heavy and light chain genes encoding phosphocholine-specific antibodies: a model for T15-idiotype dominance. J. Exp. Med. 176:1637-1643.

12. Desaymard, C., Giusti, A.M., and Scharff, M.D. 1984. Rat anti-T15 monoclonal antibodies with specificity for VH- and VH-VL epitopes. Mol. Immunol. 21:961-967.

13. Kearney, J.F., Barletta, R., Quan, Z.S., and Quintans, J. 1981. Monoclonal vs. heterogeneous anti-H-8 antibodies in the analysis of the antiphosphorylcholine response in $\mathrm{BALB} / \mathrm{c}$ mice. Eur. J. Immunol. 11:877-883.

14. Taki, S., Schmitt, M., Tarlinton, D., Feorster, I., and Rajewsky, K. 1992. T cell-dependent antibody production by Ly-1 B cells. Ann. NY Acad. Sci. 651:328-335.

15. Harnett, W., and Harnett, M.M. 1999. Phosphorylcholine: friend or foe of the immune system? Immunol. Today. 20:125-129.

16. Ylä-Herttuala, S., et al. 1994. Rabbit and human atherosclerotic lesions contain IgG that recognizes epitopes of oxidized LDL. Arterioscler.
Thromb. 14:32-40.

17. Kantor, A.B., Merrill, C.E., Herzenberg, L.A., and Hillson, J.L. 1997. An unbiased analysis of $\mathrm{V}(\mathrm{H})-\mathrm{D}-\mathrm{J}(\mathrm{H})$ sequences from B-1a, B-1b, and conventional B cells. J. Immunol. 158:1175-1186.

18. Briles, D.E., Forman, C., Hudak, S., and Claflin, J.L. 1982. Anti-phosphorylcholine antibodies of the T15 idiotype are optimally protective against Streptococcus pneumoniae. J. Exp. Med. 156:1177-1185.

19. Shan, H., Shlomchik, M., and Weigert, M. 1990. Heavy-chain class switch does not terminate somatic mutation. J. Exp. Med. 172:531-536.

20. Itabe, H., et al. 1996. Oxidized phosphatidylcholines that modify proteins. Analysis by monoclonal antibody against oxidized low density lipoprotein. J. Biol. Chem. 271:33208-33217.

21. Claflin, J.L., and Cubberley, M. 1980. Clonal nature of the immune response to phosphocholine. VII. Evidence throughout inbred mice for molecular similarities among antibodies bearing the T15 idiotype. J. Immunol. 125:551-558.

22. Sigal, N.H., Gearhart, P.J., and Klinman, N.R. 1975. The frequency of phosphorylcholine-specific B cells in conventional and germfree BALB/C mice. J. Immunol. 114:1354-1358.

23. Masmoudi, H., Mota-Santos, T., Huetz, F., Coutinho, A., and Cazenave, P.A. 1990. All T15 Id-positive antibodies (but not the majority of VHT15+ antibodies) are produced by peritoneal CD5+ B lymphocytes. Int. Immunol. 2:515-520.

24. Kantor, A.B., and Herzenberg, L.A. 1993. Origin of murine B cell lineages. Annu. Rev. Immunol. 11:501-538.

25. Pillai, S. 1999. The chosen few? Positive selection and the generation of naive B lymphocytes. Immunity. 10:493-502.

26. Hayakawa, K., et al. 1999. Positive selection of natural autoreactive B cells. Science. 285:113-116.

27. Clarke, S.H., and Arnold, L.W. 1998. B-1 cell development: evidence for an uncommitted immunoglobulin (Ig)M+ B cell precursor in B-1 cell differentiation. J. Exp. Med. 187:1325-1334.

28. Lam, K.-P., and Rajewsky, K. 1999. B cell antigen receptor specificity and surface density together determine B-1 versus B-2 cell development. J. Exp. Med. 190:471-477.

29. Segal, D.M., et al. 1974. The three-dimensional structure of a phosphorylcholine-binding mouse immunoglobulin Fab and the nature of the antigen binding site. Proc. Natl. Acad. Sci. USA. 71:4298-4302.

30. Hörkkö, S., et al. 1996. Antiphospholipid antibodies are directed against epitopes of oxidized phospholipids. Recognition of cardiolipin by monoclonal antibodies to epitopes of oxidized low density lipoprotein. J. Clin. Invest. 98:815-825.

31. Hörkkö, S., Miller, E., Branch, D.W., Palinski, W., and Witztum, J.L. 1997. The epitopes for some antiphospholipid antibodies are adducts of oxidized phospholipid and beta2 glycoprotein 1 (and other proteins). Proc. Natl. Acad. Sci. USA. 94:10356-10361.

32. Napoli, C., et al. 1997. Fatty streak formation occurs in human fetal aortas and is greatly enhanced by maternal hypercholesterolemia. Intimal accumulation of low density lipoprotein and its oxidation precede monocyte recruitment into early atherosclerotic lesions. J. Clin. Invest. 100:2680-2690. 